\title{
Allpass Delay Chain-Based IIR PR Filterbank and Its Application to Multiple Description Subband Coding
}

\author{
Bhavani Shankar M. R. and Anamitra Makur
}

\begin{abstract}
A class of infinite impulse response (IIR) perfect reconstruction (PR) filterbank is obtained with an allpass delay chain and finite impulse response (FIR) matrices at the analysis side. Such a formulation leads to a filterbank that can be optimized to any desired response. For a first-order allpass, the synthesis bank becomes FIR. Design examples showing warping of the frequency scale and filters with unequal passband widths are presented. The latter part of the work deals with designing a filterbank for a multiple description coding scenario. This is achieved in two parts: by obtaining the MMSE synthesis bank for a given analysis bank and channel state and by optimizing the analysis response to minimize the average distortion in the presence of channel erasures and quantization. The proposed IIR filterbank, as well as the wellknown FIR paraunitary filterbank, are optimized for various channels, and their performances for the two-channel case have been compared with the derived optimal performance.
\end{abstract}

Index Terms-Allpass function, IIR filterbank, multiple description coding, subband coding.

\section{INTRODUCTION}

A NUMBER of attempts have been made in obtaining infinite impulse response (IIR) filterbanks based on allpass warping or allpass delay chain. These filterbanks, which are typically nonorthogonal and have unequal passband width, have several applications such as in speech/audio. In [1], a non-perfect reconstruction (PR) warped discrete Fourier transform (DFT) filterbank is obtained by replacing the delay chain by an allpass function and using the inverse discrete Fourier transform (IDFT) polyphase matrix. In [2], a non PR unequal width filterbank is formulated by warping a uniformly modulated bank of equal passband width. A constant polyphase matrix warped DFT filterbank with PR is proposed in [3]. While earlier works are limited to non-PR solutions or constant polyphase matrices, in this work, we formulate a class of warped delay chain-based IIR PR filterbanks of any length. The delay chain is warped using the well-known allpass transformation [4] from $z$ domain to $\hat{z}$ given by

$$
\hat{z}^{-1}=A(z)
$$

where $A(z)$ is a stable allpass function.

Notable among other IIR PR filterbank works are [5]-[7]. In [5], a two-channel IIR bank with causal stable inverse is formulated. In [6], IIR biorthogonal causal stable filterbanks are char-

Manuscript received May 9, 2000; revised December 6, 2001. The associate editor coordinating the review of this paper and approving it for publication was Dr. Xiang-Gen Xia.

The authors are with the Department of Electrical Communication Engineering, Indian Institute of Science, Bangalore, India.

Publisher Item Identifier S 1053-587X(02)02394-2. acterized using unimodular matrices, but the design procedure is rather involved. In [7], an IIR bank with allpass polyphase components and IDFT matrix is formulated. The synthesis filters are anticausal stable, and a method based on time reversing is proposed to implement these banks to have PR property. The IIR PR filterbank proposed in this work, in spite of nonlinear warping, can, in principle, be optimized for any desired shape. For the case when the allpass or the polyphase obey certain constraints (as given in footnote 3), the proposed PR bank becomes a warped version of a prototype PR FIR bank. For the case of a first-order allpass and FIR matrices, we obtain a FIR synthesis bank.

In this work, we also present an application of the proposed filterbank for multiple description coding (MDC) [8]. In MDC, a source is transmitted over $M$ erasure channels ${ }^{1}$ with a rate constraint, resulting in some distortion. Such erasure channels may occur in a packet network, where error detection is present but no retransmission is permitted. The receiver must obtain an estimate of the lost packets from the available ones. The aim is to minimize the average distortion

$$
D=\sum_{s_{i} \in(0,1)} p_{\left(s_{0}, s_{1} \cdots s_{M-1}\right)} D_{\left(s_{0}, s_{1} \cdots s_{M-1}\right)}
$$

where $s_{0}, s_{1} \cdots s_{M-1}$ denotes the state of various channels such that $s_{i}=1$ if the $i$ th channel is received and $s_{i}=0$ if it is not received. $D_{\left(s_{0}, s_{1} \cdots s_{M-1}\right)}$ is the minimum mean square distortion for the state $\left(s_{0}, s_{1} \cdots s_{M-1}\right)$, and $p_{\left(s_{0}, s_{1} \cdots s_{M-1}\right)}$ is the channel state probability.

For quantizers, multiple description scalar quantization has been proposed in [9]. While source coders such as transform and subband coders perform decorrelation, MDC necessitates the transmitted descriptions of the source to be correlated. Toward this goal, multiple description transform coding introduces a correlating transform [10], [11]. Both consider $M=2$ channel case with fine quantization assumption, a fixed rate constraint, and mean square estimation at the receiver. In [12], multiple description sub-band coding is introduced for a specific case. The authors consider unconstrained filterbanks, only an $M=2$ channel case, and a specific channel with equal probability of erasure. Further, they assume fine quantization and use the theoretical rate-distortion bound to obtain the optimal filterbank for a maximum permissible distortion constraint.

We determine the minimum mean square estimator receiver under the uncorrelated additive quantization noise assumption. For a given rate constraint, and for the optimum receiver, we obtain an expression for the average distortion. The bit alloca-

\footnotetext{
${ }^{1}$ Channels in which the message is either received correctly or not received at all.
} 


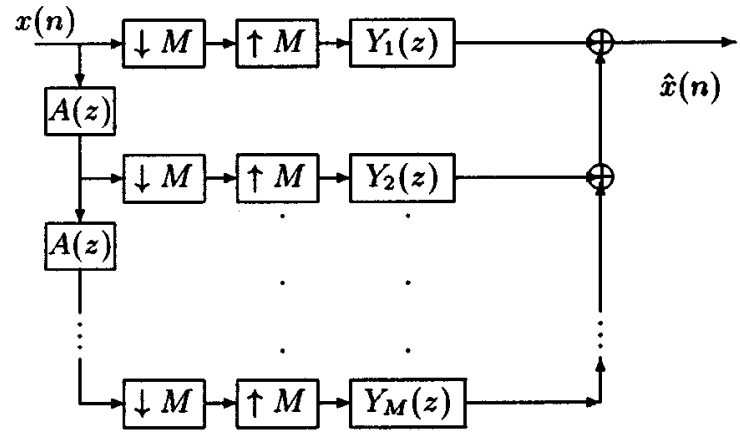

Fig. 1. Warped PR delay chain system.

tion is assumed to be for no channel loss. The result is for any number of channels and for any state probability set. This result allows us to design a filterbank minimizing the average distortion. We present results of such designs for various channels, various sources, and paraunitary as well as biorthogonal (proposed) banks. These results for the two-channel case are compared with the derived minimum possible average distortion.

\section{IIR PR BANK WITH WARPED DELAY CHAIN}

\section{A. PR Warped Delay Chain}

It is trivial to show that an $M$-channel delay chain, along with the downsamplers and upsamplers, is PR. Now, consider the warping scheme of (1) at the analysis side. Let us start with the structure shown in Fig. 1, where the filters $Y_{i}(z), 1 \leq i \leq M$ are used to achieve PR. Using the alias component (AC) matrix $\mathbf{H}(z)$ [13], the PR requirement becomes

$$
\mathbf{H}(z) \underline{Y}^{T}(z)=[M, 0, \ldots 0]^{T}
$$

where $\underline{Y}(z)=\left[Y_{1}(z), Y_{2}(z), \ldots Y_{M}(z)\right]$. The AC matrix in the case of the allpass delay chain is a Vandermonde matrix

$$
\begin{aligned}
& \mathbf{H}(z) \\
& \quad=\left(\begin{array}{ccccc}
1 & A(z) & A^{2}(z) & \cdot & A^{M-1}(z) \\
1 & A(z W) & A^{2}(z W) & \cdot & A^{M-1}(z W) \\
1 & A\left(z W^{2}\right) & A^{2}\left(z W^{2}\right) & \cdot & A^{M-1}\left(z W^{2}\right) \\
& & \ddots & & \\
1 & A\left(z W^{M-1}\right) & A^{2}\left(z W^{M-1}\right) & \cdot & A^{M-1}\left(z W^{M-1}\right)
\end{array}\right)
\end{aligned}
$$

where $W=e^{-j 2 \pi / M}$. A unique solution exists if the determinant of $\mathbf{H}(z)$ is nonzero on the unit circle. From the form of the determinant of Vandermonde matrix [14], we see that unique solution exists iff $A\left(z W^{j}\right)$ are distinct for $0 \leq j \leq M-1$ on the unit circle. The requirements on $A(z)$ such that this is true are mentioned later.

The solution $\underline{Y}(z)$ of (3) is the first column of $\mathbf{H}^{-1}(z)$ [14], such that $Y_{i}(z)$ is the co-efficient of $x^{i-1}$ in the polynomial

$$
T(x)=M \frac{\prod_{i=1}^{M-1}\left(x-A\left(z W^{i}\right)\right)}{\prod_{i=1}^{M-1}\left(A(z)-A\left(z W^{i}\right)\right)} .
$$

For instance

$$
\begin{aligned}
Y_{M}(z) & =1 / D(z) \\
Y_{M-1}(z) & =-\sum_{i=1}^{M-1} A\left(z W^{i}\right) / D(z) \\
Y_{M-2}(z) & =\sum_{i=1}^{M-1} \sum_{j>i}^{M-1} A\left(z W^{i}\right) A\left(z W^{j}\right) / D(z)
\end{aligned}
$$

and so on, where

$$
D(z)=\frac{1}{M} \prod_{i=1}^{M-1}\left(A(z)-A\left(z W^{i}\right)\right)
$$

Since $1 / D(z)$ is common to all branches, it can be moved after the add chain and implemented in cascade. This reduces the computation and is expected to be more robust to co-efficient quantization. If $A(z)$ is causal stable, the synthesis filters remaining before the add chain $\underline{Y}(z) D(z)$ are causal stable. However, $1 / D(z)$ is causal stable iff $D(z)$ is minimum phase. ${ }^{2}$ This is, however, not true in general. For such cases, $D(z)$ can be split into minimum and maximum phase systems and $1 / D(z)$ realized, as in [7]. Since it is a delay-free PR solution, requisite amount of delay needs to be added to $1 / D(z)$. The synthesis filters are, in general, IIR. However, for first-order allpass, the synthesis filters are FIR [15]. All the filters have real coefficients if $A(z)$ has real coefficients [15].

\section{B. Requirements on $A(z)$}

Let $A(z)$ be a $K$ th-order allpass function of the form

$$
A(z)=e^{j \alpha_{0}} \prod_{k=1}^{K} \frac{1-a_{k}^{*} z}{z-a_{k}}
$$

where $a_{k}=\alpha_{k} e^{-j \beta_{k}}, 1 \leq k \leq K$ are its poles. Since $A(z)$ is causal stable, $\left|a_{k}\right|<1 \forall k . \mathbf{H}(z)$ is invertible iff $A\left(z W^{j}\right)$ are distinct for $0 \leq j \leq M-1$. The distinctness requirement is met by forcing the phase of $A(z)$ to be monotone decreasing in the range $[-\pi, \pi]$.

Noncausal Phase Compensation: For first-order allpass, since its phase is monotone decreasing and spans $[-\pi, \pi]$, both constraints are automatically satisfied, but the phase of a $K$ th-order causal stable allpass is monotone decreasing and spans a range $[-K \pi, K \pi]$ [13]. This range is reduced to $[-\pi, \pi]$ using a noncausal delay [1], $\hat{A}(z)=z^{(K-1)} A(z)$. The delay chain is warped using $\hat{A}(z)$ and not $A(z)$. $\hat{A}(z)$ is not causal; therefore, its phase is not monotone, although it spans $[-\pi, \pi]$.

Monotone Phase: An allpass may be designed imposing monotone phase as follows. The phase is monotone if the group delay is positive (monotone decreasing) or negative (monotone increasing). While phase of $A(z)$ is monotone decreasing in $[-K \pi, K \pi]$, the phase of $z^{(K-1)}$ is monotone increasing in $[-(K-1) \pi,(K-1) \pi]$. Therefore, the phase of $\hat{A}(z)$ cannot

${ }^{2} D(z)$ has poles inside the unit circle when $A(z)$ is stable. 
be monotone increasing for all $\omega$, and so, the group delay of $\hat{A}(z)$, given by [4]

$$
\operatorname{grd}(\hat{A}(\omega))=1-K+\sum_{k=1}^{K}\left(\frac{1-\alpha_{k}^{2}}{1-2 \alpha_{k} \cos \left(\omega+\beta_{k}\right)+\alpha_{k}^{2}}\right)
$$

should be positive [1]

$$
\operatorname{grd}(\hat{A}(\omega))>0 \quad \forall \omega .
$$

The poles need to be chosen such that (7) is satisfied at the minimum of $\operatorname{grd}(\hat{A}(\omega))$. The requirement of real coefficients leads to the poles occurring in conjugate pairs. Consider a complex pole pair $\alpha_{i} e^{ \pm j \beta_{i}}$ for some $i$. It can be shown that the minima of (7) depends on $\beta_{i}$. Further, it can be shown that positive real poles $\left(\beta_{i}=0\right)$ and complex pole pairs with $0<\beta_{i}<$ $\pi / 2$ have minima at $\pi$. Moreover, negative real poles $\left(\beta_{i}=\pi\right)$ and complex pole pairs with $\pi / 2<\beta_{i}<\pi$ have minima at 0 . The imaginary poles $\left(\beta_{i}=\pi / 2\right)$ have minima at 0 and $\pi$. Therefore, we can group the poles in two classes based on the location of their minima. The constraint of (7) for each of such classes can be written as

$$
\sum_{k}\left(\frac{1-\alpha_{k}^{2}}{1+\alpha_{k}^{2}+2 \alpha_{k}\left|\cos \left(\beta_{k}\right)\right|}\right)>K-1
$$

where $k$ runs over the number of poles in the class.

It is difficult to find the minimum of $\operatorname{grd}(\hat{A}(\omega))$ and, hence, a constraint on the poles for a general case. Instead, the poles are grouped into the two classes mentioned previously, and the poles of each class are chosen to satisfy the corresponding constraints [as given in (8)]. Since the minimum of the overall group delay is greater than the minima for isolated classes added together, this approach satisfies (7). However, the classification of the poles need to be decided a priori. Example 1 (and continued later) illustrates the use of such constraint in designing a filterbank.

Example 1: To design a second-order allpass with conjugate imaginary pole pairs $a_{1}, a_{2}=\alpha_{1} e^{ \pm j \pi / 2}$, the constraint of (7), using (8) with $\beta_{k}=\pi / 2$, becomes $0<\alpha_{1}<1 / \sqrt{3}$.

\section{PR Banks}

Having derived the PR solution for a warped delay chain, we now extend it to a general filterbank. Consider a PR polyphase matrix pair so that $\mathbf{R}(z) \mathbf{E}(z)=z^{-n_{0}} \mathbf{I} \forall z$, where $n_{0}$ depends on the length of the filters used, and $\mathbf{I}$ is an $M \times M$ identity matrix. With such a pair, it is easy to show that the system in Fig. 2 is PR. The matrices can either be FIR or IIR. We choose FIR matrices since it is easier to obtain FIR polyphase matrices satisfying the aforesaid condition than IIR polyphase matrices [6]. It, therefore, results in a filterbank structure where the delay chain at the analysis side is IIR with the matrix after downsamplers being FIR. ${ }^{3}$ With chosen matrices $\mathbf{E}(z), \mathbf{R}(z)$, the analysis fil-

\footnotetext{
${ }^{3}$ It is possible to use this structure to warp a starting FIR bank with polyphases $\mathbf{E}^{\prime}, \mathbf{R}^{\prime}$ if either $\mathbf{E}^{\prime}$ or $\mathbf{R}^{\prime}$ are scalar, or $A(z)$ is a function of $z^{M}$. In both cases, warping $\mathbf{E}^{\prime}\left(z^{M}\right)$ using $\hat{A}(z)$ results in a function of $z^{M}$, which can be moved across the downsamplers to obtain a system like Fig. 2. For the second case, resulting $\mathbf{E}(z)$ will be IIR, and PR is achieved with $\hat{A}^{-\eta_{0} M}\left(z^{1 / M}\right) \mathbf{R}^{\prime}\left(\hat{A}^{-M}\left(z^{1 / M}\right)\right)$.
}

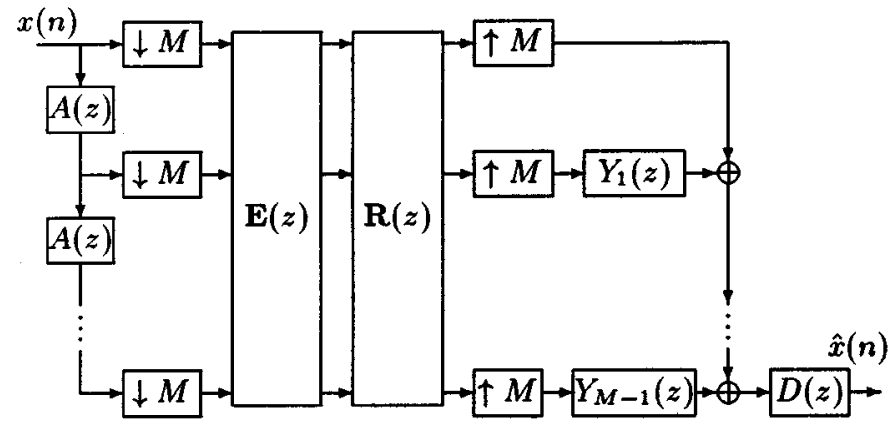

Fig. 2. PR filterbank with warped delay chain. (a)

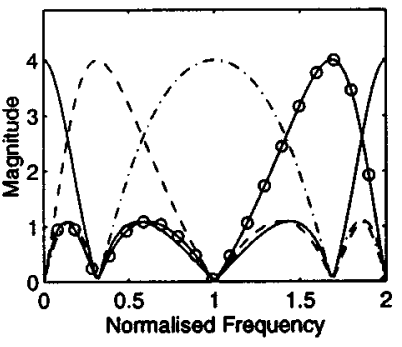

(b)

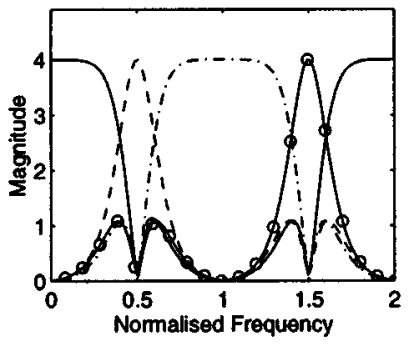

Fig. 3. Proposed analysis filterbanks with DFT $\mathbf{E}(z), \mathbf{R}(z)$; four channel. (a) First-order warping $a_{1}=0.3$. (b) Second-order warping of Example $1 a_{1}=$ $0.55 j, a_{2}=a_{1}^{*}$.

ters $\underline{H}(z)=\left[H_{0}(z), H_{1}(z) \ldots, H_{M-1}(z)\right]^{T}$ and the synthesis filters $\underline{F}(z)=\left[F_{0}(z), F_{1}(z) \ldots, F_{M-1}(z)\right]^{T}$ are obtained as

$$
\begin{aligned}
\underline{H}(z) & =\mathbf{E}\left(z^{M}\right) \underline{A}^{T}(z) \\
\underline{F}^{T}(z) & =\underline{Y}(z) \mathbf{R}\left(z^{M}\right)
\end{aligned}
$$

where $\underline{A}(z)=\left[1, A(z) \cdots A^{M-1}(z)\right]$.

From earlier mentioned properties, we obtain a PR IIR filterbank. For first-order allpass, the analysis bank is causal stable IIR, whereas the synthesis bank is FIR. If the matrix $\mathbf{E}(z)$ is paraunitary, then the resulting analysis filters $\underline{H}(z)$ are power complementary. It should be noted that the synthesis filters are power complementary with paraunitary $\mathbf{R}(z)$ only when the allpass poles are zero.

\section{Design Results}

DFT Banks: $\mathbf{E}(z)$ is chosen to be the $M \times M$ IDFT matrix $\mathbf{W}^{*}$ and its PR pair $\mathbf{R}(z)$ is $\mathbf{W}$. This formulation leads to a warped DFT filterbank. No optimization is involved in this case, except for the delay chain allpass poles. Fig. 3(a) shows a fourchannel example with first order warping $a_{1}=0.3$.

Example 1 (Continued): The prototype four-channel DFT filterbank has crossover frequencies $\omega_{0}=0.25 \pi$ and $\omega_{1}=$ $0.75 \pi$ [such that $\left|H_{i}\left(\omega_{i}\right)\right|=\left|H_{i+1}\left(\omega_{i}\right)\right|$ in the transition band]. A bank is designed so that the crossover frequencies are at $0.4 \pi$ and $0.6 \pi$. A warping function using the earlier second-order allpass, which results in this frequency transformation and satisfies the earlier constraint, is $\alpha_{1}=0.55$. The resulting filter responses are shown in Fig. 3(b).

General Banks: In the ensuing designs, $\mathbf{E}(z)$ and $\mathbf{R}(z)$ are chosen to be FIR paraunitary (of length 8 ) because of efficient design and ease of achieving PR. For the two-channel case, lat- 

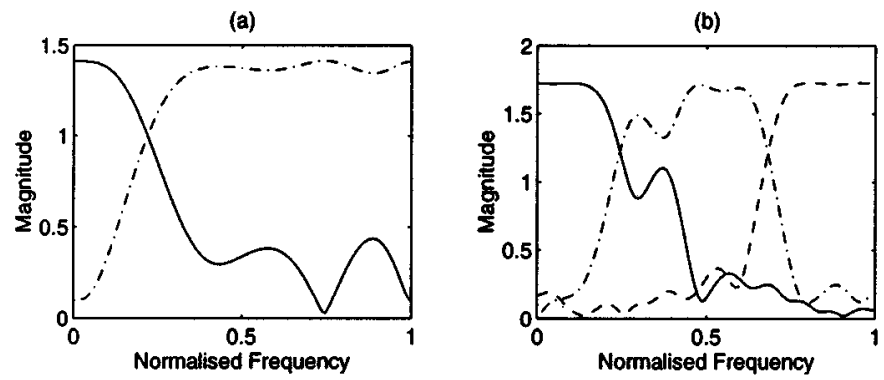

Fig. 4. Proposed analysis filterbanks with paraunitary $\mathbf{E}(z), \mathbf{R}(z)$ of length 8. First-order warping. (a) Two-channel, cutoff $\pi / 3$, optimized $a_{1}=-0.6109$. (b) Three-channel, cutoff $[\pi / 4,2 \pi / 3]$, optimized $a_{1}=0.1696$.

tice structure [13] is used, whereas the householder factorization [13] is used for a three-channel bank to obtain $\mathbf{E}(z) . A(z)$ is taken as a first-order allpass with real pole. The resulting analysis filter responses are calculated using (9). The responses are minimized only for the stopband energies (since the analysis filters are power complementary) using a joint optimization of $A(z)$ and $\mathbf{E}(z)$. Examples of filter responses with various cutoffs along with the resulting values of $a$ are shown in Fig. 4 with relevant parameters given in the caption. These examples are for unequal passband width filterbanks.

Some earlier formulations of unequal width filterbanks use unequal integer or rational resampling factors [16], [17]. Moreover, most of the designs in [16] and [17] result in analysis filters of unequal lengths. For the case of proposed filterbanks, resampling factors are the same in all the subbands. Moreover, equal length $\mathbf{E}(z)$ results in analysis filters having equal orders for the proposed banks.

\section{Optimum Multiple Description SubBAND CODER}

We now turn our attention toward designing a filterbank minimizing the average distortion, given in (2), for the MDC scenario. The first step is to obtain the optimum synthesis bank for a given analysis bank and a given channel state and to evaluate the corresponding MSE distortion $D_{\left(s_{0}, s_{1}, \ldots, s_{M-1}\right)}$.

\section{A. Optimum Synthesis Filters}

Consider an $M$-channel subband coding scenario, the filterbank being PR and the channels being of erasure type. Assuming the additive noise model for the quantizers, let $\eta_{i}(n)$ and $\sigma_{\eta_{i}}^{2}$ be the quantization noise and its variance on the $i$ th channel.

Fig. 5(a) depicts the scenario with only $K$ subbands received, where $K \leq M$. (Without loss of generality, we assume that the first $K$ bands are available.) Our goal is to provide an optimum estimate of the output $\hat{x}(n)$ and obtain an expression for the minimum output error variance.

Referring to Fig. 5(a), after the synthesis polyphase matrixing, the channel ordering is lost, and the outputs are time ordered. Therefore, we require $M$ such outputs for an $M$-channel critically sampled bank, the reason for $\mathbf{A}(\omega)$ being an $M \times K$ matrix. Due to $M$-fold upsamplers, $\hat{x}(n)$ is cyclo wide sense stationary (CWSS) with a period $M$, and a direct application of the Wiener solution is not applicable on scalar $\hat{x}(n)$. However, $\hat{x}(n M-i)=v_{i}(n) 0 \leq i \leq M-1$, and the vector $\underline{v}(n)=\left[v_{0}(n), v_{1}(n) \cdots v_{M-1}(n)\right]^{T}$ is WSS. Let

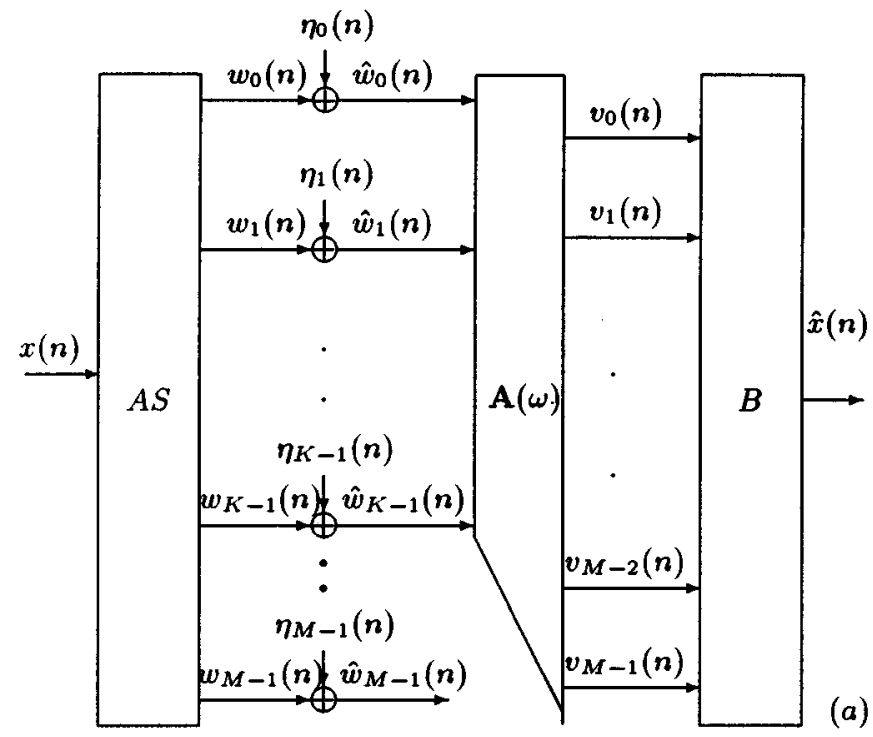

(a)

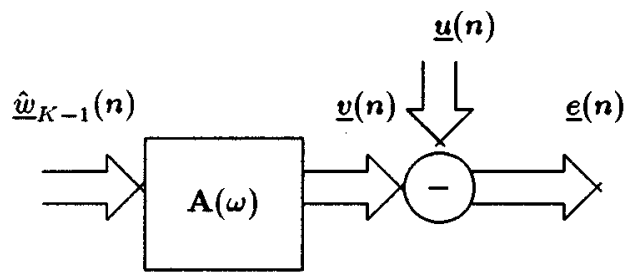

(b)

Fig. 5. (a) Subband coder (AS: analysis, B: upsample, delay, and add). (b) Equivalent matrix Wiener filter.

the output vector of $\mathbf{R}(\omega)$ (PR synthesis without quantization) be $\underline{u}(n)$. The vector $\underline{u}(n)$ is WSS and, due to PR, $\underline{u}(n)$ is the same as the input vector to the analysis side. Therefore, the matrix Wiener solution can be obtained to minimize the variance of the blocked error $\underline{e}(n)=\underline{v}(n)-\underline{u}(n)$. Define $\underline{w}_{i}(n)=\left[w_{0}(n), w_{1}(n) \cdots w_{i-1}(n)\right]^{T}$ and $\underline{\hat{w}}_{i}(n)=\left[\hat{w}_{0}(n), \hat{w}_{1}(n) \cdots \hat{w}_{i-1}(n)\right]^{T}$ for some $i$, where $w_{j}$ and $\hat{w}_{j}$ are the unquantized and quantized $j$ th sub-band signals, as shown in Fig. 5(a). The matrix Wiener solution [18] that minimizes $E\left[\underline{e}^{T}(n) \underline{e}(n)\right]$ in Fig. 5(b) is given by

$$
\mathbf{A}(\omega)=\mathbf{S}_{\underline{\underline{w}}_{K-1} \underline{u}^{\mathbf{H}}}^{\mathbf{H}}(\omega) \mathbf{S}_{\underline{\hat{w}}_{K-1}}^{-1} \underline{\hat{w}}_{K-1}(\omega)
$$

where $\mathbf{H}$ denotes Hermitian operation, and $\mathbf{S}_{\underline{a b}}(\omega)$ is the auto/cross power spectral density matrix of vector processes $\underline{a}(n)$ and $\underline{b}(n)$. Since $\underline{u}(n)$ is obtained by filtering $\underline{w}_{M-1}(n)$ with $\mathbf{R}(\omega), \mathbf{S}_{\underline{\hat{w}}_{K-1} \underline{u}}(\omega)=\mathbf{S}_{\underline{\hat{w}}_{K-1} \underline{w}_{M-1}}(\omega) \mathbf{R}^{\mathbf{H}}(\omega)$. Assuming the quantization noise in each channel is uncorrelated with all the sub-band signals and other noise sources, it can be shown that

$$
\begin{aligned}
& \mathbf{S}_{\underline{\hat{w}}_{K-1} \underline{\hat{w}}_{K-1}}(\omega)=\mathbf{S}_{\underline{w}_{K-1} \underline{w}_{K-1}}+\mathbf{Q} \\
& \mathbf{S}_{\underline{\hat{w}}_{K-1} \underline{w}_{M-1}}(\omega)=\mathbf{S}_{\underline{w}_{K-1} \underline{w}_{M-1}}(\omega)
\end{aligned}
$$

where $\mathbf{Q}=\operatorname{diag}\left[\sigma_{\eta_{0}}^{2}, \sigma_{\eta_{1}}^{2} \cdots \sigma_{\eta_{K-1}}^{2}\right]$ is the quantization noise psd. Therefore, $\mathbf{A}(\omega)$ from (10) simplifies to

$$
\mathbf{A}(\omega)=\mathbf{R}(\omega) \mathbf{S}_{\underline{w}_{K-1}}^{\mathbf{H}} \underline{w}_{M-1}(\omega) \mathbf{S}_{\underline{\hat{w}}_{K-1}}^{-1} \underline{\hat{w}}_{K-1}(\omega) .
$$


Continuing further, the MMSE $D_{(1 \cdots 1,0 \cdots 0)}$ can be shown to be equal to

$$
\begin{aligned}
E\left[x^{2}(n)\right]- & \frac{1}{2 \pi M} \int_{0}^{2 \pi} \\
& \cdot \operatorname{trace}\left(\mathbf{A}(\omega) \mathbf{S}_{\underline{\hat{w}}_{K-1} \underline{\hat{w}}_{K-1}}(\omega) \mathbf{A}^{\mathbf{H}}(\omega)\right) d \omega .
\end{aligned}
$$

One curious observation is that for any input whose psd is $M$ fold symmetric, and for any PR bank, the MMSE of (14) is independent of the filters used in the absence of quantization [15].

\section{B. Design of Analysis Filters}

The analysis side uses a single analysis bank since erasures are not known a priori. However, erasures are known to the receiver. Therefore, the synthesis side switches to the optimum $\mathbf{A}(\omega)$ for the occurred channel state for a given data segment. Therefore, repeatedly using (14) to find the distortion for each possible channel state on the right-hand side of (2), an expression for the average distortion $D$ is obtained for the given analysis bank. We now proceed with the design by optimization of the analysis filterbank to minimize $D$ for a given channel probability set $\left\{p_{\left(s_{0}, s_{1} \cdots s_{M-1}\right)}\right\}$ and for a given input power spectral density.

The quantization error in each band depends on the bit allocation. Since the distortion occurs due to channel erasures as well as due to quantization, the optimal bit allocation should minimize (2) (given a filterbank). However, it is difficult to get the closed-form solution for the same. Therefore, an inner iterative bit allocation optimization should be done along with analysis bank optimization. To avoid this, we use a fixed bit allocation that is optimal for subband coding [19]. Such an allocation is nearly optimal for MDC scenario where the contribution of the states involving some lost channels toward the average distortion $D$ are not affected much by the bit allocation (e.g., high bit-rate) or not significant enough themselves [e.g., $p_{(1 \cdots 1)} D_{(1 \cdots 1)}$ dominates $D$ ]. In our work, fractional and negative bit allocations are not treated separately. Moreover, the optimal synthesis filters are unconstrained. Optimum FIR synthesis filters are obtained for FIR analysis bank in [20] for a different context. There is no FIR restriction on the analysis bank in our work. Optimum FIR synthesis solution for the present scenario may be found in [21].

1) Simplified Channel Probabilities: The minimization of (2) is not affected by any positive scaling. In addition, the term $D_{(0 \cdots 0)}$ is independent of the filterbank used and plays no role in the optimization. Scaled and simplified cost functions are used in our simulations, which are described below.

For $M$ channels, the distortion of (2) will have $2^{M}-1$ nonredundant terms, with $M+1$ terms arising out of none or only one channel being lost. The other terms denote two or more channels being lost simultaneously. Assuming that probability of more than one channel failing simultaneously is insignificant, the cost function is dominated by single channel erasure and quantization distortions. Therefore, the distortion may be approximated as

$$
D=\sum_{i \in(0, M-1)} p_{i} D_{i}+p_{(1 \cdots 1)} D_{(1 \cdots 1)}
$$

TABLE I

Performance of MDC-OPtimized Two-ChanNel Proposed Filterbanks FOR AR(1) SOURCE WITH $\rho=0.5$ FOR VARIOUS BIT RATES

\begin{tabular}{c|c|c}
\hline$R$ & $\bar{D}_{\text {channel }}$ & $\bar{D}_{\text {quant }}$ \\
\hline 1 & 0.0209 & 0.1483 \\
2 & 0.0215 & 0.0445 \\
3 & 0.0213 & 0.0117 \\
4 & 0.0212 & 0.0029 \\
5 & 0.0210 & $7.55 e-4$ \\
6 & 0.0210 & $1.89 e-4$ \\
7 & 0.0210 & $4.50 e-5$ \\
8 & 0.0208 & $1.04 e-5$ \\
\hline
\end{tabular}

where $p_{i}=p_{\left(s_{0}, s_{1} \ldots, s_{M-1}\right)}$, and $D_{i}=D_{\left(s_{0}, s_{1} \ldots, s_{M-1}\right)}$ of (2) with $s_{j}=1, j \neq i, 0 \leq j \leq(M-1)$, and $s_{i}=0$. In addition, the $p_{i}$ s and $p_{(1 \cdots 1)}$ are scaled so that their sum is unity. For the two-channel case, three scaled probability terms $\left[p_{(1,1)}, p_{(0,1)}, p_{(1,0)}\right]$ form the probability set. For the threechannel case, the scaled probability set with the aforesaid simplifications is $\left[p_{(1,1,1)}, p_{0}, p_{1}, p_{2}\right]$. These probability sets are used to quantify the channel in further treatment.

\section{Results}

We report results for both the paraunitary and the biorthogonal class. While any PR filterbank can be used for this design, two- and three-channel FIR paraunitary banks and proposed IIR biorthogonal banks with first-order allpass are optimized for various channels and source statistics. The parameters for optimization are $\mathbf{E}(z)$ for a paraunitary bank, whereas $\mathbf{E}(z)$ and allpass pole $a$ are used for the proposed bank. $\mathbf{E}(z)$ is chosen to be the FIR paraunitary matrix using the householder factorization [13] for these cases. Moreover, the designed banks will be referred as MDC-optimized banks in the tables and figure captions.

Effect of Bit Rate on Distortion: We study the effect of bit rate on the average distortion for a given source and a channel. The distortion of (14) can be expressed as the sum of channel distortion and quantization distortion components, as given in Appendix A. We define $\bar{D}_{\text {quant }}$ as the average quantization distortion [obtained from second term of (19)] for all channel states and $\bar{D}_{\text {channel }}$ in a similar fashion such that $D=\bar{D}_{\text {quant }}+$ $\bar{D}_{\text {channel. }}$ It is observed that while $\bar{D}_{\text {quant }}$ has an exponential decay with bit rate, $\bar{D}_{\text {channel }}$ depends on the channel and the optimized filters. With bit-rate variation, since the optimized filters are different, $\bar{D}_{\text {channel }}$ varies slightly.

Such behavior is illustrated in the examples below. The twochannel model used is $[1-\alpha, \alpha, 0]$. The results given in Table I for the channel with $\alpha=0.1$ for a two-channel proposed filterbank with $\operatorname{AR}(1)$ source $(\rho=0.5)$ are representative, and similar results and observations can be obtained for other channels. Fig. 6 shows a few responses of the proposed banks for an $\operatorname{AR}(1)$ source $(\rho=0.5)$ for various bit rates. The bank changes from having equal passband filters (good sub-band coder) to one with unequal passband filters. Note that both $\bar{D}_{\text {quant }}$ and $\bar{D}_{\text {channel }}$ contribute to $D$ as well as filter shapes at sufficiently low bit rates. However, only $\bar{D}_{\text {channel }}$ affects $D$ and shapes at sufficiently high bit rates. Consequently, we now present results separately for low and high rates. ${ }^{4}$

\footnotetext{
${ }^{4}$ The exact bit rate at which the significance of the contribution from $\bar{D}_{\text {quant }}$ and $\bar{D}_{\text {channel }}$ to $D$ varies is dependent on the channel. We have omitted the singular case wherein $\bar{D}_{\text {channel }}$ is zero from the above discussion.
} 

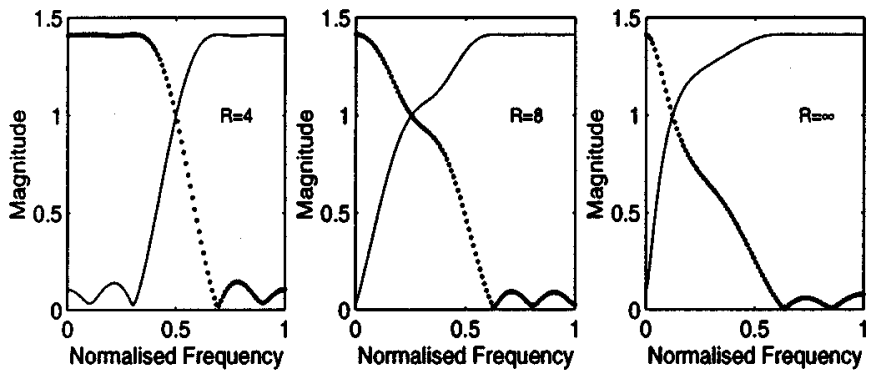

Fig. 6. MDC-optimized proposed filters of Table I, where the length of $\mathbf{E}(z)$ is 6 .

Low Bit Rates: From (21), $D_{\text {channel }}$ for a given channel state is reduced by increasing the correlation among the available and lost channels $(\mathbf{Y}(\omega))$ and/or reducing the variance in the lost channels $\left(\mathbf{S}_{\text {lost }}(\omega)\right)$. On the contrary, $D_{\text {quant }}$ increases with increased correlation among any bands since decorrelation is necessary for good sub-band coding [also seen from equation (18)]. Generalizing to the average of all channel states, a tradeoff is attained between channel distortion and quantization distortion during optimization. For variance preserving filters, at low correlation, the emphasis is on minimization of the variance in the channels with high erasure probabilities so that both distortions can be brought down. The following results illustrate these facts.

The results are reported for a fixed bit rate $(R=1)$ for various sources and various channels. Table II gives the performance of two-channel proposed and paraunitary filterbanks for AR(1) source ( $\rho=0.5$ ) with the distortions for paraunitary banks superscripted by $P U$. The earlier channel model is used with various values of $\alpha$. Fig. 7 shows the response of the proposed filters for $\alpha=0$ and $\alpha=0.01$.

At $\alpha=0, D_{(0,1)}$ does not contribute to $D$ and, hence, can take higher values, as seen from Table II. It can be seen from Fig. 7 that the first filter passes a larger signal variance. Its loss would result in a larger error, which is offset by a zero weighting. Only $D_{(1,1)}$ needs to be minimized, and the filters are optimized for subband coding. It is observed that the value of the allpass pole of the proposed bank $a_{o p t}$ is nearly zero for these filters. Therefore, these filters are nearly variance preserving. For $\alpha=$ 0.01 , we have a nonzero contribution from $D_{(0,1)}$. Thus, both $D_{(0,1)}$ and $D_{(1,1)}$ need to be minimized. There is a change in the filter shapes (approximately swapping), as shown in Fig. 7. This reduces $D_{(0,1)}$ as the first filter now passes lower signal variance. Since the filter shapes almost remain the same (except for the swapping), $D_{(1,1)}$ remains nearly the same as the earlier minimized value. Further, it is observed that for higher values of $\alpha$, both $D_{(0,1)}$ and $D_{(1,1)}$, remain nearly the same.

Three-channel proposed filterbanks for AR(2) source with $r=0.75, \theta=\pi / 2$ are shown here in Fig. 8 with the channel model $[1-\alpha, 0, \alpha, 0]$.

High Bit-Rates: The following are the results for high bit rates $\left(\sigma_{\eta_{i}}^{2} \rightarrow 0\right)$. Since $\bar{D}_{\text {quant }}$ is negligible, only $\bar{D}_{\text {channel }}$ needs to be minimized. Further, since the bank is assumed PR when all the channels are present, $p_{(1 \cdots 1)}$ is taken as $x$, whose value plays no role in the optimization. " $x$ " is taken as zero in our simulations. As mentioned earlier, average distortion is reduced by increasing the correlation among the channels and
TABLE II

PERFormance OF MDC-Optimized Two-Channel Proposed FilterbanKs FOR AR(1) SOURCE WITH $\rho=0.5$ FOR VARIOUS CHANNELS WITH $R=1$

\begin{tabular}{c|c|c|c|c|c|c}
\hline$\alpha$ & $D_{(0,1)}$ & $D_{(0,1)}^{P U}$ & $D_{(1,1)}$ & $D_{(1,1)}^{P O}$ & $D$ & $D^{P U}$ \\
\hline 0 & 0.8540 & 0.8570 & 0.1550 & 0.1551 & 0.1550 & 0.1551 \\
0.01 & 0.2971 & 0.2972 & 0.1551 & 0.1551 & 0.1565 & 0.1565 \\
0.1 & 0.2968 & 0.2971 & 0.1551 & 0.1551 & 0.1692 & 0.1692 \\
0.5 & 0.2964 & 0.2971 & 0.1552 & 0.1551 & 0.2258 & 0.2262 \\
1 & 0.2964 & 0.2971 & 0.1554 & 0.1551 & 0.2964 & 0.2971 \\
\hline
\end{tabular}
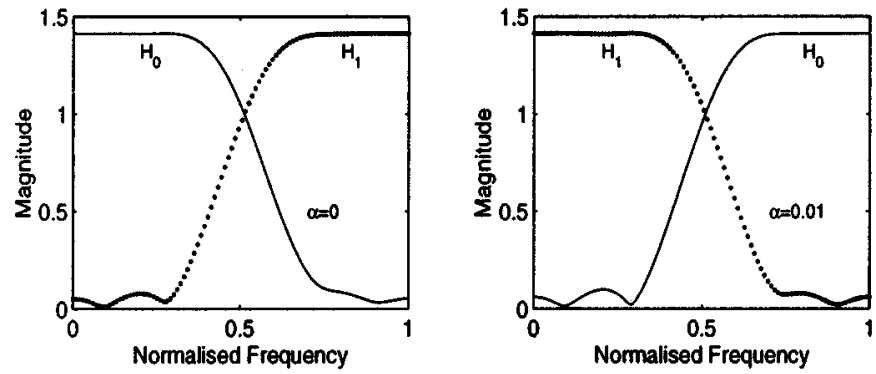

Fig. 7. MDC-optimized proposed filters of Table II, where the length of $\mathbf{E}(z)$ is 6 , and $R=1$.
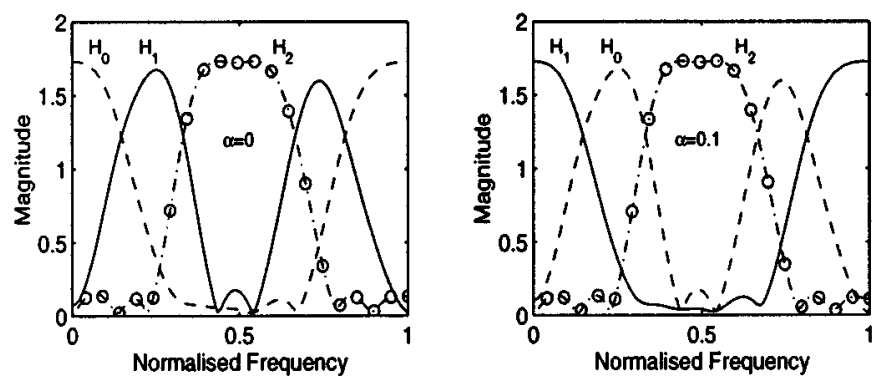

Fig. 8. MDC-optimized three-channel proposed filters for AR(2) source with $r=0.75, \theta=\pi / 2$, where the length of $\mathbf{E}(z)$ is 6 , and $R=1$.

reducing the signal variance in the channels with high erasure probability.

For two channels, the channel model used is $[x, 1-\alpha, \alpha]$, and results are listed in Table III for various values of $\alpha$ for the proposed and paraunitary filterbanks for various sources. In order to compare our optimized design, we also present the minimum possible average distortion $D_{o p t}$ in each case. In Appendix B, a simplified expression for $D_{\text {channel }}$ is derived for single erasure. This result is further used in Appendix $\mathrm{C}$ to find the analytic solution for $D_{o p t}^{P U}$ in the case of two channels (as well as the unconstrained optimal paraunitary banks). In addition, following the approach of [12], $D_{\text {opt }}$ for two-channel biorthogonal banks is determined in this Appendix. The unconstrained optimal biorthogonal bank shapes can be obtained from [12], which turn out to have singular polyphase.

It is observed from Table III that the designed paraunitary banks perform close to optimal paraunitary banks. However, performance of optimal biorthogonal banks are expectedly superior to that of paraunitary banks (except at $\alpha=0$ ). The designed biorthogonal banks using the proposed method perform close to optimal biorthogonal banks at $\alpha=0$. The difference between performance of designed and optimal biorthogonal banks arise due to the fact that optimal biorthogonal banks have a polyphase component with pole at every $\omega$ [12], which is 
TABLE III

Optimal AND ObTAINed PerformanCE OF MDC-Optimized 2 ChanNel FILTER BANKS AT High BIT RATE

\begin{tabular}{c|c|c|c|c|c|c}
\hline Source & $\alpha$ & $a_{\text {opt }}$ & $D$ & $D^{P U}$ & $D_{\text {opt }}$ & $D_{\text {opt }}^{P U}$ \\
\hline AR(1), & 0 & -0.4214 & 0.2080 & 0.2081 & 0.2048 & 0.2048 \\
$\rho=0.5$ & $1 / 4$ & -0.9061 & 0.2723 & 0.2823 & 0.2048 & 0.2799 \\
& $1 / 2$ & -0.8720 & 0.2666 & 0.3000 & 0.2048 & 0.3000 \\
\hline AR(2), & 0 & -0.6954 & 0.1399 & 0.1421 & 0.1379 & 0.1379 \\
$r=0.75$, & $1 / 4$ & -0.7573 & 0.1801 & 0.2176 & 0.1379 & 0.2153 \\
$\theta=\pi / 4$ & $1 / 2$ & -0.8870 & 0.1931 & 0.2307 & 0.1379 & 0.2307 \\
\hline
\end{tabular}

infeasible to imitate using first-order polyphase IIR filterbank. For larger $\alpha$, the performance of proposed banks is consistently better than not only the designed but the optimal paraunitary banks as well.

For three channels, the channel model used is $[x, 1-\alpha-$ $\beta, \alpha, \beta]$, and similar results are listed in Table IV for various values of $\alpha$ and $\beta$. The $D_{o p t}^{P U}$ for three channels with $\alpha=\beta=$ 0 can be obtained from the derivations in Appendix B. Their values for the AR(1) and AR(2) sources mentioned in Table IV are 0.1210 and 0.1492 , respectively. It is seen that the designed paraunitary banks perform close to optimal for $\alpha=\beta=0$. Filter responses of two-channel paraunitary and three-channel proposed banks are shown in Figs. 9 and 10. Note how the first filter of Fig. 9, for example, changes from highpass at $\alpha=0$ to allpass (delay) at $\alpha=0.5$ (to lowpass at $\alpha=1$ which is not shown). Similarly, since the second and the third channels are error free in Fig. 10, these filters have nearly the same passband at the high energy part of the spectrum, whereas the first filter occupies the low energy spectrum.

A few comments and observations are in order. Source spectra that are $M$-fold symmetric are not considered for reasons stated earlier. The distortion of (2) is invariant to permutations of the weighting functions and the associated distortions, and therefore, the associated filter responses are also invariant. For example, in the two-channel case, the filterbank optimized for $1-\alpha$ is the same as the filterbank optimized for $\alpha$ with the filters interchanged. This is why we consider $\alpha$ in the range 0 to 0.5 in Table III. It can be seen that for $\alpha=0.5$, the paraunitary filters are allpass (delays), which is a result that is obtained in [12]. It is observed that the average distortion increases as the weighting functions tend to identical values. Comparing the result for $\alpha=1$ in Table II and $\alpha=0$ (which is the same for $\alpha=1$ if filters are swapped), we see that $D$ has reduced from 0.2964 to 0.2080 , which shows the significance of quantization.

It is worthwhile to mention about the performance of other banks vis-a-vis the obtained banks. For a two-channel delay chain with $\alpha=0.5$, the average distortion for $\mathrm{AR}(1)$ and $\mathrm{AR}(2)$ sources in Table III is 0.3 and 0.2307 , respectively. The distortion for a three-channel delay chain with $\alpha=\beta=1 / 3$ for the AR(1) and AR(2) sources in Table IV is 0.2 and 0.2530 , respectively. Hence, for equally likely erasure channels $(\alpha=1 / 2$ for two-channel and $\alpha=\beta=1 / 3$ for three-channel), the average distortion for the delay chain is nearly the same as that of the paraunitary bank (and almost same as that of the proposed bank). In comparison, for the two-channel case and $\operatorname{AR}(1)$ source $(\rho=0.5)$, the average distortion for a $2 \times 2$ Karhunen-Loeve transform and a length 20 paraunitary bank designed for minimum stopband energy is 0.4472 and 0.4928 ,
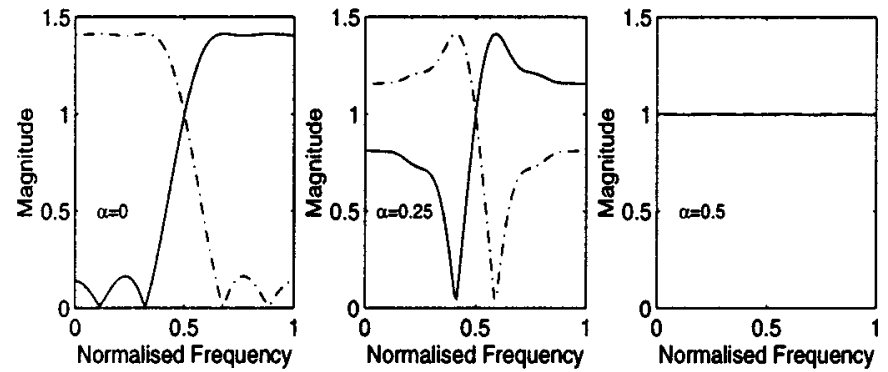

Fig. 9. MDC-optimized two-channel paraunitary filters of Table III for AR(1) source, where the length of $\mathbf{E}(z)$ is 6 .
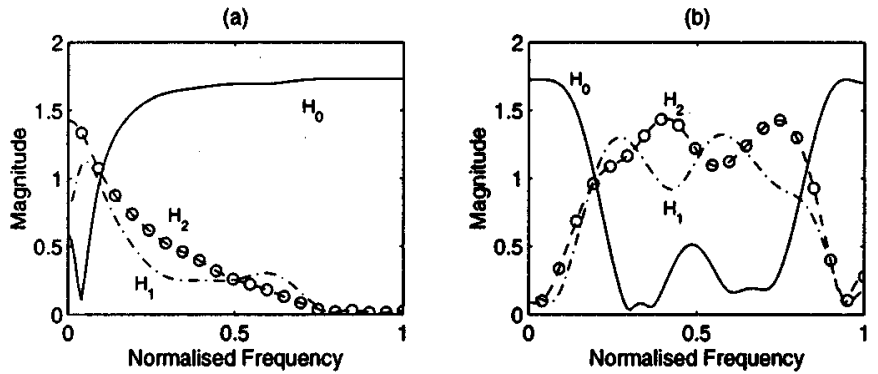

Fig. 10. MDC-optimized three-channel proposed filters of Table IV for $\alpha=$ $\beta=0$, where the length of $\mathbf{E}(z)$ is 6 . (a) $\mathrm{AR}(1)$ source. (b) $\mathrm{AR}(2)$ source.

TABLE IV

Performance of MDC-Optimized Three-ChanNel Filter BANKS AT HIGH BIT RATE

\begin{tabular}{c|c|c|c|c}
\hline Source & $\alpha, \beta$ & $a_{\text {opt }}$ & $D$ & $D^{P U}$ \\
\hline & 0,0 & 0.7971 & 0.1216 & 0.1219 \\
$\mathrm{AR}(1)$, & $0.2,0.4$ & -0.3270 & 0.1838 & 0.1953 \\
$\rho=0.5$ & $1 / 3,1 / 3$ & -0.2081 & 0.1968 & 0.2 \\
& $0.1,0.8$ & -0.3737 & 0.1486 & 0.1573 \\
\hline & 0,0 & -0.1113 & 0.1530 & 0.1531 \\
$\operatorname{AR}(2)$, & $0.2,0.4$ & -0.6223 & 0.2060 & 0.2472 \\
$\theta=\pi / 2, \mathrm{r}=0.75$ & $1 / 3,1 / 3$ & -0.6047 & 0.2200 & 0.2530 \\
& $0.1,0.8$ & 0.4870 & 0.1816 & 0.1995 \\
\hline
\end{tabular}

respectively. Comparing these with the average distortions for the designed banks (Table III), we see that the designed filterbanks are better performing since they achieve an average distortion of 0.2666 (for the proposed bank) and 0.3 (for the paraunitary bank). While the delay chain system turns out to be a very good candidate for symmetric channels, it does not perform well compared with the designed banks for asymmetric channel probabilities.

\section{CONCLUSION}

The proposed IIR PR filterbank, even though it is natural for warped frequency scale, can be designed for any shape. Due to monotone phase requirement, the allpass poles should be designed to satisfy certain conditions. However, for first-order allpass, there is no constraint, and the synthesis bank becomes FIR. Design examples illustrate cases involving warping of frequency scale and involving unequal passband widths with a uniform resampling factor. 
For a multiple-description coding scenario, the optimum unconstrained synthesis filters are obtained using uncorrelated additive noise model of the quantizers, as well as using a fixed bit allocation optimal for fine quantization and no erasures. Using the MMSE synthesis banks, the analysis bank is optimized to minimize average distortion. Bit allocation strategy and quantization noise modeling provide the scope of further work toward the optimum MDC filterbank. Performances for optimal MDC banks for the two-channel case are derived, and the performance of the designed banks compare favorably with the optimal performance.

Several interesting conclusions have been drawn from MDCoptimized filterbank examples. While quantization distortion (negative exponentially related to rate) dominates at low rates, channel distortion takes over at high rates. As a result, at low rates, the filterbank has nearly equal passband width, is nearly variance preserving, where the warping parameter $a_{o p t}$ is nearly 0 . A subband with higher erasure probability will occupy the low energy part of the spectrum, sometimes with a noncontiguous passband. On the other hand, at high rates, the filter shapes are determined by the channel. Based on the channel probability, a filter transforms from highpass to allpass to lowpass. For symmetric channels, since filters tend to be allpass, the delay chain system performs very well. This, fortunately, is not true for asymmetric channels. It is also observed that the average distortion becomes higher as a channel changes from highly asymmetric to symmetric, which is consistent with the capacity of the channel.

\section{APPENDIX A}

\section{CHANNEL AND QUANTIZATION DisTORTION}

Here, we express the distortion of (14) in two components. Putting (13) in (14), the operand under trace() reduces to

$$
\begin{aligned}
\mathbf{R}(\omega) \mathbf{S}_{\underline{w}_{K-1}}^{\mathbf{H}} & \underline{w}_{M-1}(\omega) \mathbf{S}_{\underline{\underline{w}}_{K-1}}^{-1} \underline{\hat{w}}_{K-1}(\omega) \mathbf{S}_{\underline{\hat{w}}_{K-1} \underline{\hat{w}}_{K-1}}(\omega) \\
& \times \mathbf{S}_{\underline{\hat{w}}_{K-1} \mathbf{H}} \underline{\hat{w}}_{K-1}(\omega) \mathbf{S}_{\underline{w}_{K-1} \underline{w}_{M-1}}(\omega) \mathbf{R}^{\mathbf{H}}(\omega)
\end{aligned}
$$

which simplifies to

$\mathbf{R}(\omega) \mathbf{S}_{\underline{w}_{K-1} \underline{w}_{M-1}}^{\mathbf{H}}(\omega) \mathbf{S}_{\underline{\hat{w}}_{K-1}}^{-1} \underline{\hat{w}}_{K-1}(\omega) \mathbf{S}_{\underline{w}_{K-1} \underline{w}_{M-1}}(\omega) \mathbf{R}^{\mathbf{H}}(\omega)$

since auto psd is Hermitian symmetric. From (11) and the matrix inversion lemma, we see that

$$
\begin{aligned}
\mathbf{S}_{\underline{\hat{w}}_{K-1} \underline{\hat{w}}_{K-1}}^{-1}(\omega)=\mathbf{S}_{\underline{w}_{K-1} \underline{w}_{K-1}}^{-1}(\omega)-\mathbf{S}_{\underline{w}_{K-1} \underline{w}_{K-1}}^{-1}(\omega) \\
\cdot\left[\mathbf{I}_{K}+\mathbf{S}_{\underline{w}_{K-1} \underline{w}_{K-1}}(\omega) \mathbf{Q}^{-1}\right]^{-1}
\end{aligned}
$$

where $\mathbf{I}_{K}$ is the $K \times K$ identity matrix.

It can be easily seen from the definition of the psd matrices that $\mathbf{S}_{\underline{w}_{K-1} \underline{w}_{K-1}}^{-1}(\omega) \mathbf{S}_{\underline{w}_{K-1} \underline{w}_{M-1}}(\omega)$ equals $\left[\mathbf{I}_{K}, \mathbf{S}_{\underline{w}_{K-1} \underline{w}_{K-1}}^{-1}(\omega) \overline{\mathbf{Y}}(\omega)\right]$, where $\mathbf{Y}(\omega)$ is the submatrix of $\underline{\mathbf{S}}_{K-1} \underline{w}_{M-1}(\omega)$ containing the first $K$ rows and last
$M-K$ columns. Using (17) and using the linearity of the trace operator, (16) becomes

$$
\begin{aligned}
& D_{(1 \cdots 1,0 \cdots 0)} \\
&=E\left[x^{2}(n)\right]-\frac{1}{2 \pi M} \int_{0}^{2 \pi} \\
& \cdot \operatorname{trace}[ \mathbf{R}(\omega) \mathbf{S}_{\underline{w}_{K-1} \underline{w}_{M-1}}^{\mathbf{H}}(\omega) \\
& \cdot {\left.\left[\mathbf{I}_{K}, \mathbf{S}_{\underline{w}_{K-1} \underline{w}_{K-1}}^{-\mathbf{1}}(\omega) \mathbf{Y}(\omega)\right] \mathbf{R} \mathbf{R}^{\mathbf{H}}\right](\omega) d \omega+\frac{1}{2 \pi M} \int_{0}^{2 \pi} } \\
& \cdot \operatorname{trace} {\left[\mathbf{R}^{\mathbf{H}}(\omega) \mathbf{R}(\omega)\left[\mathbf{I}_{K}, \mathbf{S}_{\underline{w}_{K-1} \underline{w}_{K-1}}^{-\mathbf{1}}(\omega) \mathbf{Y}(\omega)\right]\right.} \\
& \cdot\left[\mathbf{S}_{\underline{w}_{K-1} \underline{w}_{K-1}}^{-1}(\omega)+\mathbf{Q}^{-1}\right]^{-1} \\
&\left.\cdot\left[\mathbf{I}_{K}, \mathbf{S}_{\underline{w}_{K-1} \underline{w}_{K-1}}^{-1}(\omega) \mathbf{Y}(\omega)\right]\right] d \omega .
\end{aligned}
$$

This can be written as

$$
D_{(1 \cdots 1,0 \cdots 0)}=D_{\text {channel }}+D_{\text {quant }}
$$

where $D_{\text {channel }}$ refers to the first two terms in (18). While $D_{\text {channel }}$ contains distortion only due to the channel erasures, $D_{\text {quant }}$ is the contribution of the quantization noise to the distortion. It is simple to see that in the presence of all channels, $D_{\text {channel }}$ is zero, whereas $D_{\text {quant }}$ is zero in the absence of quantization noise. Moreover, we see that the matrices are positive semi-definite, and hence, the contribution of each of the terms is positive.

\section{APPENDIX B}

\section{DISTORTION FOR SINGLE ERASURE}

For a single channel lost and no quantization, we derive an expression for $D_{(1 \cdots 1,0)}$. Using

$$
\begin{aligned}
E\left[x^{2}(n)\right]= & \frac{1}{2 \pi M} \int_{0}^{2 \pi} \\
& \cdot \operatorname{trace}\left[\mathbf{R}(\omega) \mathbf{S}_{\underline{w}_{M-1} \underline{w}_{M-1}}(\omega) \mathbf{R}^{\mathbf{H}}(\omega)\right] d \omega
\end{aligned}
$$

and the fact that $\mathbf{S}_{\underline{w}_{K-1} \underline{w}_{M-1}}^{\mathbf{H}}(\omega)=\mathbf{S}_{\underline{w}_{M-1} \underline{w}_{M-1}}(\omega)$ $\left[\mathbf{I}_{K}, \mathbf{0}_{K \times(M-K)}\right]^{T}$ and employing the linearity of trace(), $D_{\text {channel }}$ from (18) can be written as in (21), shown at the top of the next page, where $\mathbf{S}_{\text {lost }}(\omega)$ contains the last $(M-K)$ rows and columns of $\mathbf{S}_{\underline{w}_{M-1} \underline{w}_{M-1}}(\omega)$, denoting the psd of the lost channels. Let the columns of $\mathbf{Y}(\omega)$ be denoted by $\hat{Y}_{i}(\omega)$. For the case of single channel erasure $(K=M-1)$, the expression reduces to

$$
D_{\text {channel }}=\frac{1}{2 \pi M} \int_{0}^{2 \pi} c_{M-1}(\omega) E_{M-1}(\omega) d \omega
$$

where $E_{M-1}(\omega)=S_{w_{M-1} w_{M-1}}(\omega)-\hat{Y}_{0}^{\mathbf{H}}(\omega) \mathbf{S}_{\underline{w}_{M-2} \underline{w}_{M-2}}^{-1}(\omega)$ $\hat{Y}_{0}(\omega)$, and $c_{i}(\omega)=\sum_{j=0}^{M-1}\left|\mathbf{R}_{j i}(\omega)\right|^{2}$ is the norm of the $i$ th 


$$
D_{\text {channel }}=\frac{1}{2 \pi M} \int_{0}^{2 \pi} \operatorname{trace}\left\{\mathbf{R}(\omega)\left[\begin{array}{cc}
\mathbf{0}_{K \times K} & \mathbf{0}_{K \times(M-K)} \\
\mathbf{0}_{(M-K) \times K} & \mathbf{S}_{\text {lost }}(\omega)-\mathbf{Y}^{\mathbf{H}}(\omega) \mathbf{S}_{\underline{w}_{K-1} \underline{w}_{K-1}}^{-1}(\omega) \mathbf{Y}(\omega)
\end{array}\right] \mathbf{R}^{H}(\omega)\right\} d \omega
$$

channel PR synthesis filter. Further, we see that the following matrix equation is valid:

$$
\begin{aligned}
& \left(\begin{array}{cc}
\mathbf{S}_{\underline{w}_{M-2} \underline{w}_{M-2}}(\omega) & \mathbf{0}_{M-1 \times 1} \\
\mathbf{Y}^{\mathbf{H}}(\omega) & 1
\end{array}\right)\left(\begin{array}{c}
\mathbf{S}_{\underline{w}_{M-2} \underline{w}_{M-2}}^{-1}(\omega) \mathbf{Y}(\omega) \\
E_{M-1}(\omega)
\end{array}\right) \\
& =\left[S_{w_{0} w_{M-1}}(\omega), S_{w_{1} w_{M-1}}(\omega), \cdots S_{w_{M-1} w_{M-1}}(\omega)\right]^{T}
\end{aligned}
$$

since $\mathbf{Y}(\omega)$ is indeed $\left[S_{w_{0} w_{M-1}}(\omega), S_{w_{1} w_{M-1}}(\omega), \cdots\right.$ $\left.S_{w_{M-2} w_{M-1}}(\omega)\right]^{T}$. Solving $E_{M-1}(\omega)$ using Cramer's rule and using the same in (22), we get

$D_{(1 \cdots 1,0)}=\frac{1}{2 \pi M} \int_{0}^{2 \pi} c_{M-1}(\omega) \frac{\operatorname{det}\left(\mathbf{S}_{\underline{w}_{M-1} \underline{w}_{M-1}}(\omega)\right)}{\operatorname{det}\left(\mathbf{S}_{\underline{w}_{M-2} \underline{w}_{M-2}}(\omega)\right)} d \omega$.

\section{APPENDIX C}

\section{MiNIMUM DisTORTION FOR Two CHANNELS}

Using the above result, we now find the minimum possible distortion $D_{\text {opt }}$ for two-channel paraunitary and biorthogonal filterbanks.

For paraunitary filterbanks, $c_{M-1}(\omega)=1$, and $\operatorname{det}\left(\mathbf{S}_{\underline{w}_{M-1} \underline{w}_{M-1}}(\omega)\right)$ is independent of the filterbanks used. Therefore, minimizing $D_{(1 \cdots 1,0)}$ is equivalent to maximizing $\operatorname{det}\left(\mathbf{S}_{\underline{w}_{M-2} \underline{w}_{M-2}}(\omega)\right)$. Since the determinant of a positive semi-definite matrix is maximized when the matrix is diagonal and from known results in subband coding, the optimal analysis bank is the principal component filterbank. The minimum possible $D_{(1 \cdots 1,0)}$ is

$$
D_{(1 \cdots 1,0) \circ p t}=\frac{1}{2 \pi M} \int_{0}^{2 \pi} \lambda_{\min }(\omega) d \omega
$$

where $\lambda_{\min }(\omega)$ is the smallest eigenvalue of the input psd matrix. Referring to channel model $[x, \alpha, 1-\alpha]$, if $\alpha=1$, minimizing $D$ is equivalent to minimizing $D_{(0,1)}$; therefore, minimum possible average distortion for paraunitary case, given by $D_{o p t}^{P U}$, may be obtained from (25). On similar lines, we see that for the three-channel model $[x, 1-\alpha-\beta, \alpha, \beta], D_{\text {opt }}^{P U}$ can be obtained from (25) for $\alpha=\beta=0$. When $\alpha \neq 1$, the average distortion of (2) involves $D_{(1,0)}$ and $D_{(0,1)}$ (no quantization); therefore, $D$ equals

$$
\frac{1}{4 \pi} \int_{0}^{2 \pi} \operatorname{det}\left(\mathbf{S}_{\underline{w}_{M-1} \underline{w}_{M-1}}(\omega)\right)\left[\frac{\alpha}{S_{w_{0} w_{0}}(\omega)}+\frac{(1-\alpha)}{S_{w_{1} w_{1}}(\omega)}\right] d \omega .
$$

Let trace $\left(\mathbf{S}_{\underline{w}_{M-1} \underline{w}_{M-1}}(\omega)\right)=C(\omega)$. Because of the paraunitary nature of the analysis bank, $C(\omega)$ is independent of the filters used. Differentiating (26) w.r.t either $S_{w_{1} w_{1}}(\omega)$ or $S_{w_{0} w_{0}}(\omega), D_{o p t}^{P U}$ is obtained as

$$
\begin{array}{r}
D_{o p t}^{P U}=\frac{1}{4 \pi} \int_{0}^{2 \pi} \operatorname{det}\left(\mathbf{S}_{\underline{w}_{M-1} \underline{w}_{M-1}}(\omega)\right) \\
\cdot \frac{[\sqrt{\alpha}+\sqrt{(1-\alpha)}]^{2}}{C(\omega)} d \omega .
\end{array}
$$

The resulting value of $S_{w_{0} w_{0}}(\omega)$ is $C(\omega) \sqrt{\alpha} /$ $(\sqrt{\alpha}+\sqrt{(1-\alpha)})$. Using the invariance of the trace and determinant, we can find the remaining values of $\mathbf{S}_{\underline{w}_{M-1} \underline{w}_{M-1}}(\omega)$. The filter shapes can be obtained from procedure outlined in [12, eqs. 21 and 22].

For biorthogonal filterbanks, $D_{\text {opt }}$ may be obtained using an approach similar to [12] and (24). Instead of identical weights used in [12, eq. 41], we use $[1-\alpha, \alpha]$ for calculating $D$. Using similar steps [12, eqs. 49,50], the distortion obtained will be the scaled integral of

$$
a b\left[\frac{\alpha\left(1+x^{2}\right)}{a x^{2}+b}+\frac{(1-\alpha)\left(1+y^{2}\right)}{a y^{2}+b}\right]
$$

instead of [12, eq. 46], where $a, b, x, y$ are same as in [12]. $D_{\text {opt }}$ is obtained from this equation. For $\alpha$ values other than 0 and 1 , the optimum filters are of the form given in [12, eq. 61]. For the case of $\alpha=0$ or 1 , one of the variables ( $x$ or $y$ ) can be chosen such that filters are of the form [12, eq. 61]. In either case, the optimum filters retain the larger eigenvalue of the input, whereas the error is due to the lower eigenvalue.

\section{REFERENCES}

[1] M. Kappelan, B. Straub, and P. Vary, "Flexible nonuniform filterbanks using allpass transformation of multiple order," in Proc. EUSIPCO, vol. 3, Sept. 1996, pp. 1745-1748.

[2] T. Gulzow, A. Engelsberg, and U. Heute, "Comparison of a discrete wavelet transformation and a nonuniform polyphase filterbank applied to spectral-subtraction speech enhancement," Signal Process., vol. 64, pp. 5-19, 1998.

[3] A. Makur and S. K. Mitra, "Warped discrete-Fourier transform: Theory and applications," IEEE Trans. Circuits Syst. I, vol. 48, pp. 1086-1093, Sept. 2001

[4] A. V. Oppenheim and R. W. Schaffer, Discrete-Time Signal Processing. New Delhi, India: Prentice-Hall, 1992.

[5] S. M. Phoong, C. W. Kim, P. P. Vaidyanathan, and R. Ansari, "A new class of two-channel biorthogonal filterbanks and wavelet bases," IEEE Trans. Signal Processing, vol. 43, pp. 649-665, Mar. 1995.

[6] S. Basu, C. H. Chiang, and H. M. Choi, "Wavelets and perfect reconstruction subband coding with causal stable IIR filters," IEEE Trans. Circuits Syst. II, vol. 42, pp. 24-38, Jan. 1995.

[7] S. K. Mitra and C. D. Creusere, "Image coding using wavelets based on perfect reconstruction IIR filterbanks," IEEE Trans. Circuits Syst. Video Technol., vol. 6, pp. 447-458, Oct. 1996.

[8] El. Gamal and T. M. Cover, "Achievable rates for multiple descriptions," IEEE Trans. Inform. Theory, vol. 38, pp. 851-857, Nov. 1992.

[9] V. A. Vaishampayan, "Design of multiple description scalar quantizers," IEEE Trans. Inform. Theory, vol. 39, pp. 821-834, May 1993. 
[10] Y. Wang, M. T. Orchard, and A. R. Reibman, "Optimal pairwise correlating transforms for multiple description coding," in Proc. IEEE ICIP, vol. 1, 1998, pp. 679-683.

[11] V. Goyal, "Beyond traditional transform coding," Ph.D. dissertation, Univ. Calif. Berkeley, Berkeley, CA, Jan. 1999.

[12] X. Yang and K. Ramchandran, "Optimal subband filterbanks for multiple description coding," IEEE Trans. Inform. Theory, vol. 46, pp. 2477-2490, Nov. 2000.

[13] P. P. Vaidyanathan, Multirate Systems and filterbanks. Englewood Cliffs, NJ: Prentice-Hall, 1993.

[14] W. H. Press, S. A. Teukolsky, W. T. Vetterling, and B. P. Flannery, $N u$ merical Recipes in C. Cambridge, U.K.: Cambridge Univ. Press, 1992.

[15] B. Shankar M. R. "IIR perfect reconstruction filterbanks with warped delay chain and their use in design of correlating filterbanks," Masters dissertation, Dept. Elect. Commun. Eng., Indian Inst. Sci., Bangalore, India, Jan. 2000.

[16] K. Nayebi, T. P. Barnwell, and M. J. T. Smith, "Nonuniform filterbanks: A reconstruction and design theory," IEEE Trans. Signal Processing, vol. 41, pp. 1114-1127, Mar. 1993.

[17] J. Kovacevic and M. Vetterli, "Perfect reconstruction filterbanks with rational sampling factors," IEEE Trans. Signal Processing, vol. 41, pp. 2047-2066, June 1993

[18] P. P. Vaidyanathan and V. P. Sathe, "Effects of multirate systems on the statistical properties of random signals," IEEE Trans. Signal Processing, vol. 41, pp. 131-146, January 1993

[19] P. P. Vaidyanathan and A. Kirac, "Results on optimal biorthogonal filterbanks," IEEE Trans. Signal Processing, vol. 45, pp. 932-947, Aug. 1998.

[20] K. Gosse and P. Duhamel, "Perfect reconstruction versus MMSE filterbanks in source coding," IEEE Trans. Signal Processing, vol. 45, pp. 2188-2201, Sept. 1997

[21] S. Dutta, "Design of FIR MMSE synthesis filterbanks for a subband coder with erasure channels," M.S. thesis, Dept. Elect. Commun. Eng., Indian Inst. Sci., Bangalore, India, June 2000.

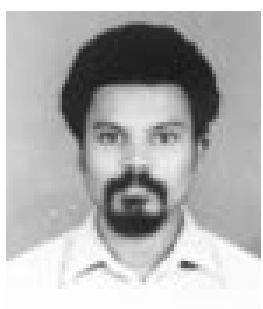

Bhavani Shankar M. R. received the B.E. degree in electronics and communication engineering from Regional Engineering College, Trichy, India, in 1998 and the M.E. degree in signal processing from the Indian Institute of Science (IISc), Bangalore, India, in 2000. He is currently pursuing the Ph.D. degree with the Department of Electrical Communication Engineering, IISc.

From 2000 to 2001, he was with SASKEN Communication Technologies, Bangalore, where he was involved with development of audio coders.

Mr. Shankar M. R. received the gold medal for academic excellence from IISc.

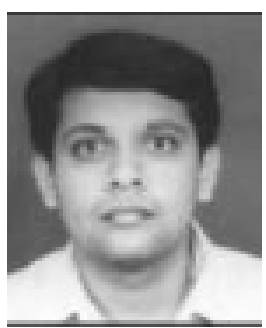

Anamitra Makur received the B.Tech. degree in electronics and electrical communication engineering from the Indian Institute of Technology (IIT), Kharagpur, in 1985 and the M.S. and Ph.D. degrees in electrical engineering from the California Institute of Technology, Pasadena, in 1986 and 1990.

$\mathrm{He}$ is currently an Associate Professor of electrical communication engineering, Indian Institute of Science (IIS), Bangalore, India. His research interests in signal compression include subband coding, filterbank design and multirate DSP, vector quantization, motion field coding, other image/video compression schemes and standards, and multimedia applications. His interests in image/video processing includes watermarking, halftoning, restoration, segmentation, and multidimensional DSP.

Dr. Makur is the recipient of the 1998 Young Engineer Award from the Indian National Academy of Engineering. 